\title{
On the Thermal Conductivity of Metals and of Insulators
}

\author{
U. Köbler \\ Research Centre Jülich, Institute PGI, 52425 Jülich, Germany \\ E-mail: u.koebler@fz-juelich.de
}

Received 25 May 2017, Accepted 16 October 2017

\begin{abstract}
The experimental indications are discussed that in insulators thermal conductivity is exclusively due to Debye bosons (sound waves). Phonons do not obviously contribute to thermal conductivity. In metals, thermal conductivity is exclusively due to electronic degrees of freedom. Phonons and Debye bosons do virtually not contribute to thermal conductivity of the metals. It appears that the electronic system of the metals has also continuum properties with bosons as excitations. We will call the bosons of the spatially continuous conduction band, CB-bosons. In contrast to the bosons of the elastic continuum (Debye bosons), CB bosons and their dispersion relation are not yet explored. Since bosons propagate ballistic, independent of lattice structure, they are the predominant carriers of thermal conductivity. Their large mean free path enables a very efficient heat transport over large distances. Identification of boson fields is limited to their heat capacities. The heat capacity of the Debye boson field is $\sim T^{3}$. The heat capacity of the CB-boson field is $\sim T$. In the approximation of an infinite mean free path of the bosons and negligible lattice contributions, thermal conductivity is proportional to the heat capacity of the boson field. Thermal conductivity therefore allows for a separate visualization of the heat capacity of the boson fields. The two power functions of temperature $\left(\sim T^{3}\right.$ and $\left.\sim T\right)$ hold up to a temperature of about $10 \ldots 30 \mathrm{~K}$ only. At this temperature thermal energy gets transferred to the atomistic degrees of freedom (phonons, band structure states). This is a typical crossover event. For larger temperatures the boson system accumulates no longer thermal energy and its heat capacity tends to zero. In this way, a sharp maximum of thermal conductivity result at about 10 to $30 \mathrm{~K}$. At ambient temperature the two power functions of temperature $\left(\sim T^{3}, \sim T\right)$ have completely disappeared. When phonons are the relevant excitations, thermal conductivity of insulators tends to zero. In metals, crossover to the conventional (atomistic) conduction band states results in a finite and nearly temperature independent thermal conductivity.
\end{abstract}

Keywords: Thermal conductivity; sound waves; boson fields.

\section{Introduction}

Thermal conductivity of solids is a not well understood phenomenon [1-3]. As is well known, in all solids heat transport can be described by the same type of differential equation [1-5]. Atomistic structures need not to be considered. This means, thermal conductivity is a macroscopic phenomenon. One could call the differential equation of heat transport universal.

As for the elastic properties of solids [6], for thermal conductivity the solid can be treated as a continuous medium. Quite generally, the excitations of a continuous medium are bosons. Independence of atomistic structures, that is universality, is typical for boson controlled thermodynamic phenomena. In fact, as we will see, the material specific atomistic system of phonons does not contribute obviously to thermal conductivity. Instead, the excitations of the continuous (elastic) solid are relevant for thermal conductivity.

The bosons of the elastic continuum are the wellknown Debye bosons (sound waves). Since the translational symmetry of the elastic continuum is invariance with respect to transformations of the length scale, the momentum of the Debye bosons is a conserved quantity. Debye bosons therefore propagate ballistic over large distances, rather independent of atomistic structures and crystal imperfections. This propagation mode can be termed universal. The ballistic propagation mode makes Debye bosons particularly suitable for heat transport. Ballistic propagation implies a simple dispersion relation. As is well known, to a good approximation the dispersion of the massless Debye bosons is a linear function of wave vector for all energies. However, the observed weak deviations from linearity (Figure 2 and 3) indicate that the Debye boson field is not a completely free field but interacts moderately with the atomistic background.

In [4] it was argued that in harmonic approximation phonons do not interact with each other and, as a consequence, are unable to transport heat. Curiously, at temperatures of 10 to $30 \mathrm{~K}$ where the harmonic approximation can be expected to hold reasonably, thermal conductivity exhibits a surprisingly sharp maximum. The sharp maximum is indicative of a crossover event, in the sense of Renormalization Group (RG) theory [7,8]. As we know from RG theory, a crossover means a change of the relevant excitation spectrum. In fact, Debye bosons and phonons are distinguished by different translation symmetries and, as a consequence, have different excitation spectra (Figures 2 and 3). Phonons are the particles of the discrete and periodic translational symmetry of the lattice while Debye bosons are the particles of the continuous 
translational symmetry of the infinite solid. The largest phonon energy is given by the inter-atomic interaction strength. Formally, for the Debye bosons with linear dispersion there is no upper energy limit. Note that the Debye boson field has identical thermodynamic properties as the electromagnetic radiation field.

Thermal energy is either in the Debye boson field or in the system of lattice vibrations. In terms of RG theory, the two thermal reservoirs get alternatively relevant. In other words, the dynamic symmetry is clearly defined by the symmetry of the relevant system. The non-relevant system accumulates no longer thermal energy and its heat capacity tends to zero. Relevance has the severe consequence that thermal population of all available states of the atomistic and of the continuous system cannot be according to the Boltzmann factor but is subject to the symmetry selection rule of relevance. For instance, at sufficiently low temperatures phonons are not relevant, and their heat capacity is zero (see Figure 4). Under this condition atomistic structures are unimportant. The observed lowtemperature heat capacity of the solid then is the heat capacity of the Debye boson field and follows $\sim \mathrm{T}^{3}$ dependence. Vanishing heat capacity contributions of phonons can be evidenced by showing that the observed heat capacity agrees with the heat capacity of the Debye boson field, calculated from the known sound velocities [9]. The $\mathrm{T}^{3}$ function of the Debye boson field is universal not so much because it is observed for all solids but because it holds over a finite temperature range, up to crossover of thermal energy to the lattice system. In the observed heat capacity, the crossover to phonon system shows up as deflection from $\mathrm{T}^{3}$ function. This is a gradual process.

As will be shown in this communication, thermal conductivity (Figure 5 and 6) provides an individual visualization of the heat capacity of the Debye boson field, at least qualitatively. This is because thermal conductivity is exclusively due to Debye bosons. Without interactions with the lattice, the heat capacity of the Debye boson field would be according to $T^{3}$ function for all temperatures. It is evident that the dramatic increase of heat capacity according to $T^{3}$ function cannot continue up to high temperatures. In fact, the $\mathrm{T}^{3}$ function stops at crossover of thermal energy to the phonon system. At ambient temperature the exploding $\mathrm{T}^{3}$ function has completely disappeared, meaning that the heat capacity of the Debye boson field is near zero. A vanishing heat capacity of the Debye boson field at elevated temperatures reveals from the fact that for all solids the observed heat capacity saturates reasonably at atomistic Dulong-Petit limit. This shows that at ambient temperature the observed heat capacity is determined by the lattice vibrations (phonons). As a consequence, the dispersion relation of the Debye bosons is no longer thermally populated and the heat capacity of the Debye boson field virtually is zero. Nevertheless, at all temperatures, excitation of Debye bosons (sound waves) is possible out of thermal equilibrium, irrespective of whether or not the dispersion of the Debye bosons is thermally populated. Heat transport by Debye bosons is also a process out of thermal equilibrium and is possible at all temperatures.

Above crossover temperature, when phonons have become the relevant excitations, the observed (total) heat capacity continuous increasing but thermal conductivity decreases dramatically. This reflects the decreasing heat capacity of the Debye boson field, and, at the same time, shows that the relevant phonons do no contribute to thermal conductivity. The sharp maximum of thermal conductivity is near crossover temperature. In the total heat capacity, the transfer of thermal energy from boson field to lattice system appears as beginning saturation towards Dulong-Petit limit.

The finite validity range of Debye's $T^{3}$ function results from the fact that the dispersion of the Debye bosons is fairly linear for all energies. Note that the dispersion of the acoustic phonons is given by sine function of wave vector and therefore is linear asymptotically for $q \rightarrow 0$ only. At elevated temperatures, the acoustic phonons become the relevant excitations because they have lower dispersion energies. Crossover to phonon system is associated with an analytical change from universal $\mathrm{T}^{3}$ function to a different and material specific function of temperature. However, this crossover proceeds in two steps (Figure 4). Below a first crossover at $\mathrm{T}_{\mathrm{AC}}$ (amplitude crossover) phonons do not contribute at all to the heat capacity. For $T<T_{A C}$, finite contributions of phonons to the heat capacity are an exceptional situation [9]. Relevant phonons are noticed by a heat capacity that is larger than the heat capacity of the Debye boson field calculated from the known sound velocities. In other words, the observed heat capacity can be only larger than the calculated Debye heat capacity. Equivalently, the calorimetric Debye temperature, evaluated from heat capacity measurements, can be only smaller than the elastic Debye temperature calculated from the sound velocities. It is evident that because of possible contributions of phonons, the correct Debye temperature has to be evaluated from sound velocity measurements and not from heat capacity measurements [9]. Note that the Debye temperature is the characteristic parameter of the boson field.

In the temperature range of $T<T_{A C}$ phonons are negligible and thermal conductivity reaches its maximum. This shows clearly that thermal conductivity is due to Debye bosons. For $T>T_{A C}$ the heat capacity of the phonon system is no longer negligible. Emerging heat capacity contributions due to phonons is not a gradual process but proceeds as a crossover event. As long as the heat capacity of the Debye bosons is larger than the emerging heat capacity of the phonons, Debye bosons remain the relevant excitations, and the universal $T^{3}$ function is preserved. The finite contribution of the non-relevant phonons is to increase the pre-factor of the $T^{3}$ function. This type of crossover we have called amplitude crossover. It is typical for finite but non-relevant energy degrees of freedom that they leave the universal class (the exponent) unchanged and enter the pre-factor of the universal power function only. The intrinsic heat capacity of the non-relevant energy degrees of freedom therefore becomes not obvious. As a conclusion, for a limited temperature interval (between $\mathrm{T}_{\mathrm{AC}}$ and a second crossover at $T^{*}$ ) phonons and Debye bosons contribute together to the observed heat capacity. This temporally increases the heat capacity over the Debye value [10]. The phonon enhanced heat capacity of the Debye boson field gives rise to a sharply decreasing Debye temperature as a function of temperature [11]. Finally, at a second crossover temperature at $T^{*}$, phonons become the relevant excitations and take over dynamics. The $T^{3}$ function of the Debye boson field then ceases and the observed heat capacity increases more slowly as a function of increasing temperature. For $T>T^{*}$ the Debye boson field accumulates no longer thermal energy, and its heat capacity tends to zero. In this way the heat capacity of the Debye 
boson field assumes an unusually sharp maximum. Thermal conductivity just reflects this behavior of the heat capacity of the Debye boson field. Note that in the limit of negligible lattice contributions and a mean free path of the Debye bosons of larger than the linear dimension of the sample, thermal conductivity is strictly proportional to the heat capacity of the Debye boson field. For $T>T^{*}$ the observed heat capacity increases much weaker than $\mathrm{T}^{3}$ function. This lets $\Theta_{D}(T)$ increase again [11]. Due to the two crossover events at $T=T_{A C}$ and at $\mathrm{T}=\mathrm{T}^{*}$ a sharp minimum results in $\Theta_{D}(T)$. Note, however, that in temperature regions with finite phonon contributions, evaluation of a Debye temperature is not meaningful. For temperatures of $T>T^{*}$ thermal conductivity of insulators already is vanishingly small and approaches zero. At ambient temperature, the residual thermal conductivity of the insulators is on the strongly decaying tail of the heat capacity of the Debye boson field. As a conclusion, when phonons come into play (for $T>T_{A C}$ ) thermal conductivity starts decreasing strongly. This shows that thermal conductivity by phonons is negligibly small. Interactions with phonons impede heat transport by Debye bosons.

Symmetries are very useful basic concepts to start with, also in thermodynamics. However, for all parts of physics symmetry violations are known. In the present context this means that in spite of their different (translational) symmetries, Debye bosons and phonons are not completely independent of each other but interact visibly. Interactions of Debye bosons with the atomistic background have two effects: they shorten the mean free path of the bosons and decrease their velocity (Figure 1) [6]. This is as for photons travelling across an optically dense medium. In fact, in all solids sound velocities decrease as a function of increasing temperature. The slope of the Debye dispersion line therefore is not perfectly constant but decreases weakly with thermal energy. Note that the sound velocity measured at temperature $T$ gives the slope of the Debye boson dispersion relation at thermal energy $k_{B} T$ [12]. If the dispersion relation of the Debye bosons is already slightly curved at the lowest temperatures this can cause marginal deviations from perfect $\mathrm{T}^{3}$ function in the heat capacity of the boson field. Very sensitive in this respect are plots of $c_{p}(T) / T^{3}$ vs. temperature. For strong Debye boson-phonon interaction no really constant behavior results for $T \rightarrow 0$ in plots of $c_{p}(T) / T^{3}[10,11]$. $\mathrm{n}$ insulators with high crystal perfection and low intrinsic Debye boson-phonon interaction, thermal conductivity starts with the $T^{3}$ function of the heat capacity of the Debye boson field (Figure 7). However, in thermal conductivity the $T^{3}$ function holds up to a much lower temperature than in heat capacity. This indicates a significant decrease of the mean free path of the Debye bosons as a function of increasing temperature. Onset of relevant damping processes for the Debye bosons is a threshold controlled crossover event and decreases the exponent of three in discrete steps. In imperfect crystals and/or in crystals with a strong intrinsic Debye bosonphonon interaction, the explicit temperature dependence of the mean free path of the Debye bosons superimposes on the $T^{3}$ function and decreases the exponent in thermal conductivity below a value of three. In addition to 3 , exponent values of 2.5 and of 2 can clearly be identified (Figure 7-9). As a consequence, the maximum of thermal conductivity depends significantly on the quality of the sample. Reliable absolute conductivity values can nearly not be given. Of course, for crystals with high lattice perfection, the maximum of thermal conductivity is fairly high.

Near amplitude crossover at $T_{A C}$ where phonons start contributing finitely to the heat capacity, thermal conductivity has already passed its maximum. Apparently phonons act as a sink for the Debye bosons. The strong damping of the Debye bosons for $T>T_{A C}$ makes the decreasing high temperature flank of thermal conductivity maximum more steep and lets the maximum appear more sharp. The maximum of thermal conductivity is near to the first crossover in the heat capacity at $\mathrm{T}_{\mathrm{AC}}$ and not at the expected maximum of the boson heat capacity near $\sim T^{*}$.

In metals, thermal conductivity also exhibits a sharp maximum in the same temperature range of 10 to $30 \mathrm{~K}$. However, heat transport in metals is exclusively due to electronic degrees of freedom [3]. Debye bosons are not involved in the heat transport of the metals as reveals from complete absence of the $T^{3}$ term. This is indicative of a sharp symmetry selection rule for the relevant carriers of heat transport.

\section{Construction of the Dispersion of the Debye Bosons}

The mass-less Debye bosons cannot be observed using inelastic neutron scattering. With inelastic neutron scattering, phonons are detected exclusively. This is a matter of the excitation process. By the microscopic process of impact of neutrons, the local atomistic degrees of freedom of phonons are excited. On the other hand, Debye bosons can be excited by macroscopic methods such as by a transmitter attached to the surface of the sample. In the atmosphere, excitation of sound waves is possible by vibrating membranes. In other words, propagation of sound waves is independent of the atomic structure of the medium. Only from sound velocity measurements the dispersion of the Debye bosons can be obtained. In fact, the sound velocity measured at temperature $\mathrm{T}$ gives the slope of the dispersion relation of the Debye bosons at thermal energy of $k_{B} T$. The applied sound frequency defines the sampling width along dispersion line. In the approximation of a temperature-independent sound velocity, the dispersion of the Debye bosons is a linear function of wave-vector for all energies. This, however, is an idealization since the Debye boson field is not a free field but interacts weakly with the atomistic background of phonons and lattice defects. Interactions with the atomistic background let sound velocities and elastic constants decrease slightly as a function of temperature (Figure 1) [6]. In other words, with increasing temperature, interactions between Debye bosons and acoustic phonons increase. The decreasing sound velocity leads to a weaker than linear dispersion of the Debye bosons as a function of excitation energy. In general, interactions between Debye bosons and acoustic phonons are stronger for longitudinal polarization compared to transverse polarization. Additionally, elastic constants and sound velocities depend somewhat on sample perfection. This precludes exact evaluations of the two quantities. Thermal conductivity therefore also depends on sample perfection.

Figure 1 visualizes the temperature dependence of the normalized sound velocities (Debye bosons) of cubic NaI ( $\mathrm{NaCl}$ structure) as a function of temperature [6]. These data apply to cube edge. In cubic systems the relation between sound velocities and elastic constants along cube edge are given by: $v_{L}=\left(c_{11} / \rho\right)^{1 / 2}$ and $v_{T}=\left(c_{44} / \rho\right)^{1 / 2}$ with $\rho$ as mass density or specific gravity $\left(\rho=3.67 \mathrm{~g} \cdot \mathrm{cm}^{-3}\right.$ for $\left.\mathrm{NaI}\right)$ and 
$c_{i j}$ as elastic constants [13]. It can be seen that $v_{L}$ has stronger temperature dependence than $v_{T}$. This is observed for the majority of all solids. In other words, sound waves with longitudinal polarization interact stronger with phonons compared to sound waves with transverse polarization. At Debye temperature of $\Theta_{D}=167 \mathrm{~K}$ [14] no anomaly is visible in the temperature dependence of the sound velocities. Note that in his field theory of 1912, P. Debye has cut the dispersion of the Debye bosons at an energy of $\Theta_{D} \cdot k_{B}$ in order to prevent thermal explosion of the heat capacity of the boson field according to $\sim \mathrm{T}^{3}$ function [15]. As we now know, this cutting procedure is physically not reasonable. At the time of Debye, crossover events were unknown. The $\mathrm{T}^{3}$ function stops because of the crossover of thermal energy from boson field to phonon system at about $10 \ldots 30 \mathrm{~K}$. In contrast to the boson excitation spectrum with no (intrinsic) upper energy limit, the phonon system has a clearly defined upper energy such that the observed heat capacity saturates at the atomistic Dulong-Petit limit. The unphysical consequence of Debye's cut-off procedure is that the solid should have no longer elastic properties for thermal energies of larger than $\Theta_{D} k_{B}$. This is obviously not correct. As is well known, solids have elastic properties for all temperatures up to melting point. This shows that the dispersion of the Debye bosons continues up to energies of $k_{B} T_{m}$ with $\mathrm{T}_{\mathrm{m}}$ as melting temperature. Equivalently, sound waves can be excited at all temperatures.

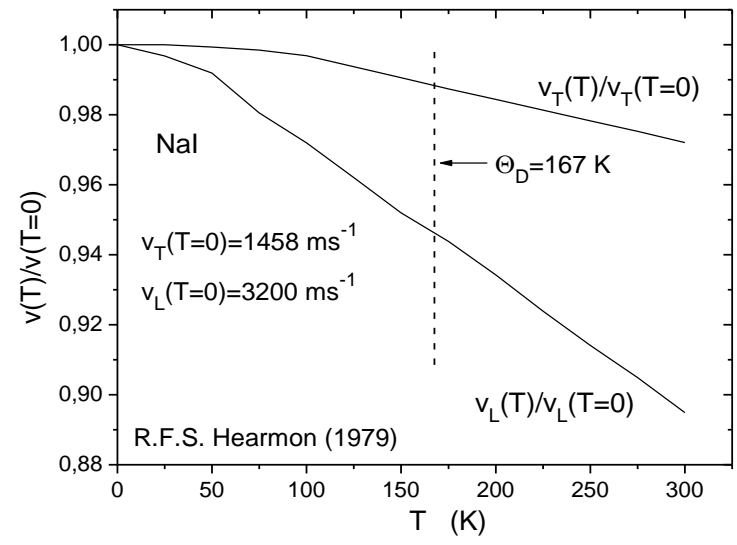

Figure 1. Normalized sound velocities for transverse polarization (upper curve) and for longitudinal polarization (lower curve) measured along cube edge of NaI as a function of temperature [6]. The stronger temperature dependence for longitudinal polarization indicates stronger interactions between sound waves (Debye bosons) and phonons. As a consequence, for longitudinal polarization the dispersion of the Debye bosons deviates stronger from linearity. No anomaly is visible at Debye temperature of $\Theta_{D}=167 \mathrm{~K}[14]$.

In order to construct the dispersion relation of the Debye bosons from the experimentally known sound velocities for transverse and longitudinal polarization $\left(v_{L / T}\right)$, we simply assume that the sound velocity measured at some temperature $\mathrm{T}, v_{L / T}(T)$, gives the slope of the dispersion of the Debye bosons at dispersion energy $E=k_{B} \cdot T$, with $\mathrm{k}_{\mathrm{B}}$ as Boltzmann constant. The corresponding reduced wave vector value is set equal to $q / q_{0}=a_{0} \cdot k_{B} T / h v_{L / T}$ with $\mathrm{a}_{0}$ as lattice parameter and h as Planck's constant. Since for most solids sound velocities and/or elastic constants have been measured up to melting temperature, it is possible to construct the dispersion of the Debye bosons up to an energy of $E=k_{B} T_{m}$ with $\mathrm{T}_{\mathrm{m}}$ as melting temperature $\left(T_{m}=933\right.$ $\mathrm{K}$ for $\mathrm{NaI})$ [16]. Commonly $k_{B} T_{m}$ is a much larger energy than the largest energy of the acoustic phonons.

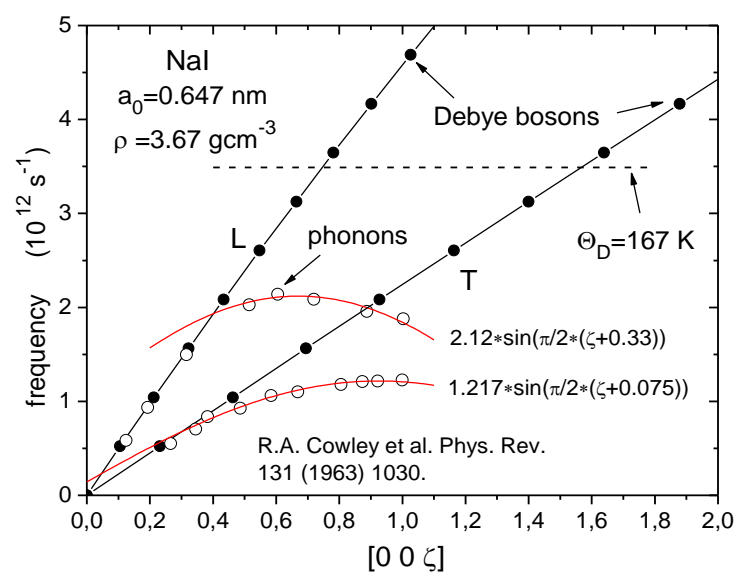

Figure 2. Dispersions of acoustic phonons with longitudinal and transverse polarization (circles), measured along cube axis $\left(a_{0}=6.4728 \AA\right.$ A) of NaI, using inelastic neutron scattering [17]. Dispersions of the Debye bosons calculated from the temperature dependence of sound velocities [6] are given by filled circles. For small wave vector values phonons have adapted to the dispersion of the Debye bosons and start linearly. No anomaly is visible at Debye's cut-off energy of $k_{B} \cdot \Theta_{D}\left(\Theta_{D}=167 \mathrm{~K}\right)$ [14].

It is a somewhat confusing experimental observation that the initially linear dispersions of the acoustic phonons agrees with the measured sound velocities. This allows evaluation of sound velocities from phonon dispersions but does not mean that Debye bosons and phonons become identical particles in the limit of large wave-lengths. The translational symmetries of the two particles remain different also for $\lambda \rightarrow \infty$. The reason for the initially identical dispersion is that due to inevitable interactions between Debye bosons and phonons the two associated dispersion relations attract each other. Note that the dispersion relations of particles with different symmetries can attract each other. The dispersions of particles with the same symmetry are repelling. For a strong Debye boson-phonon interaction the dispersion of the acoustic phonons assumes the linear dispersion of the Debye bosons over a considerable energy and wave-vector range. In other words, phonon dispersion gets attracted by the linear dispersion of the Debye bosons and not vice versa. As an example of strong Debye boson-phonon interactions Figure 2 shows phonon dispersions measured along edge of the cubic unit cell of NaI $\left(a_{0}=6.4728 \AA\right)$ using inelastic neutron scattering (circles) [17]. It can be seen that for longitudinal polarization a rather abrupt analytical crossover occurs in the dispersion relation of the acoustic phonons. Up to an energy of $\sim 2 \mathrm{THz}(\sim 96 \mathrm{~K})$ the dispersion of the acoustic phonons has adapted to the linear dispersion of the Debye bosons. The slope of the linear section agrees excellently with the sound velocity of $\sim 3200 \mathrm{~ms}^{-1}$ (filled circles) $[6,14]$. As a conclusion, phonon dispersion is attracted by the dispersion of the sound waves. Note that at $T=96 \mathrm{~K}$ the dispersion of the Debye bosons is no longer thermally populated. In other words, attraction of the dispersions of Debye bosons and acoustic phonons is independent of 
whether the dispersion relations are thermally populated or not. It is evident that the larger the linear section in the dispersion of the acoustic phonons is, the larger is the Debye boson-phonon interaction. Additionally, strong Debye boson-phonon interactions give rise to a strong temperature dependence of the sound velocity and to noticeable deviations of the dispersion of the sound waves from linearity. As a conclusion, due to Debye bosonphonon interaction it becomes possible to observe the dispersion of the mass less Debye bosons using inelastic neutron scattering.

Phonon dispersions for larger wave vector values than the linear section can be fitted by sine-functions of wave vector including a constant phase shift in the argument. As we have explained earlier [12], the empirical phase shift in the argument of the sine functions is another measure of the Debye boson-phonon interaction strength. In fact, for transverse polarization, Debye boson-phonon interaction is weaker and the phase shift is smaller. The sine function of wave vector (including phase shift) then holds over a larger q-range, and the initial section with linear dispersion is smaller. As a conclusion, strong Debye boson-phonon interactions manifest for all q-values. Note, however, that the sine function is the dispersion of the linear atomic chain [5]. This is not self-evident for three-dimensional bulk materials. It is evident that lattice theories [5] can give adequate description of phonon dispersions only in the case of negligible Debye boson-phonon interactions. In particular, a sharp functional crossover in the phonon dispersion is beyond lattice theories.

At the Debye temperature of $\mathrm{NaI}$ of $\Theta_{D}=167.6 \mathrm{~K}$ $(\approx 3.49 \mathrm{THz})$ [14] no anomaly is visible in the dispersion relations of the Debye bosons. In particular boson dispersion continues beyond Debye's cut-off limit of $\Theta_{D} \cdot k_{B}$. Another important detail in Figure 2 is that the dispersion of the Debye bosons continues much beyond zone boundary. In principle, the shortest possible wavelength of the Debye bosons is of the order of the diameter of the sources of the Debye bosons. In [12] it was argued that the sources of the Debye bosons must be the nearly spherical atomic cores.

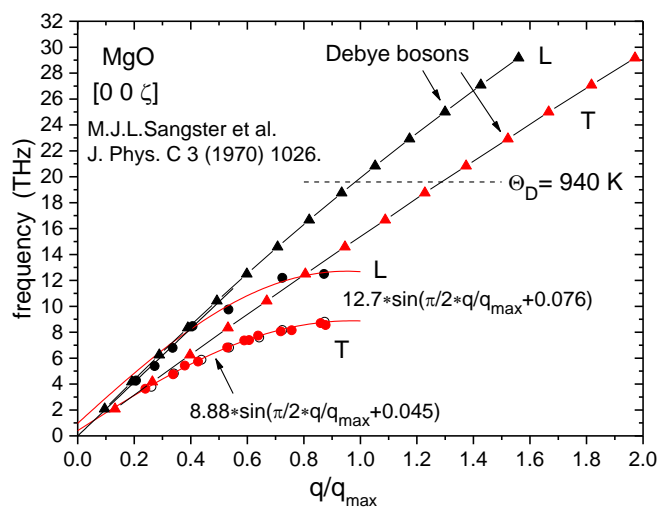

Figure 3. Dispersions of the acoustic phonons along cube edge of $\mathrm{MgO}$ (filled circles) measured by inelastic neutron scattering [18]. The dispersions of the Debye bosons have been obtained from the temperature dependence of the sound velocities (see text) [6]. Initially, phonon dispersions start with the same slope as the dispersions of the Debye bosons.
As a second example with weaker Debye bosonphonon interactions Figure 3 shows similar results as for $\mathrm{NaI}$ but for cubic $\mathrm{MgO}[6,19]$. The dispersions of the acoustic phonons approach sine functions of wave-vector more closely. The phase shifts in the argument of the sine functions are smaller than for NaI. Again, no anomaly is visible in the dispersions of the Debye bosons at $\Theta_{D} \cdot k_{B}$ with $\Theta_{D}=940 \mathrm{~K}(\sim 19.6 \mathrm{THz})$ [19]. Melting temperature of $\mathrm{MgO}$ is $T_{m}=3105 \mathrm{~K}(\sim 64.7 \mathrm{THz})$ [16]. The dispersions of the Debye bosons much continue beyond zone boundary.

Our main interest is in the question: how do phonons and Debye bosons contribute to thermal conductivity. As we have explained, thermal conductivity and heat capacity cannot be discussed independent of each other. In particular, the different appearance of the crossover of thermal energy from Debye bosons to phonons in heat capacity and in thermal conductivity has to be discussed. In the heat capacity this crossover proceeds in two steps. Below crossover temperature $\mathrm{T}_{\mathrm{AC}}$ phonons do not contribute at all to the heat capacity. This can be evidence by showing that the observed $\mathrm{T}^{3}$ function agrees with the $\mathrm{T}^{3}$ function of the Debye boson field, calculated from the observed sound velocities [9]. Interestingly, in insulators thermal conductivity has its maximum for $\mathrm{T}<\mathrm{T}_{\mathrm{AC}}$. This shows that phonons are not involved in thermal conductivity. Only for $\mathrm{T}>\mathrm{T}_{\mathrm{AC}}$ phonons contribute finitely to the heat capacity, and thermal conductivity decreases strongly. In order to illustrate the sharp onset of phonon contributions to the heat capacity Figure 4 shows data of the coefficient of linear thermal expansion, $\alpha$ [21], and of heat capacity [22] as a function of absolute temperature to a power of three. The two data sets have been matched together. The perfect proportionality conforms to Grüneisen relation.

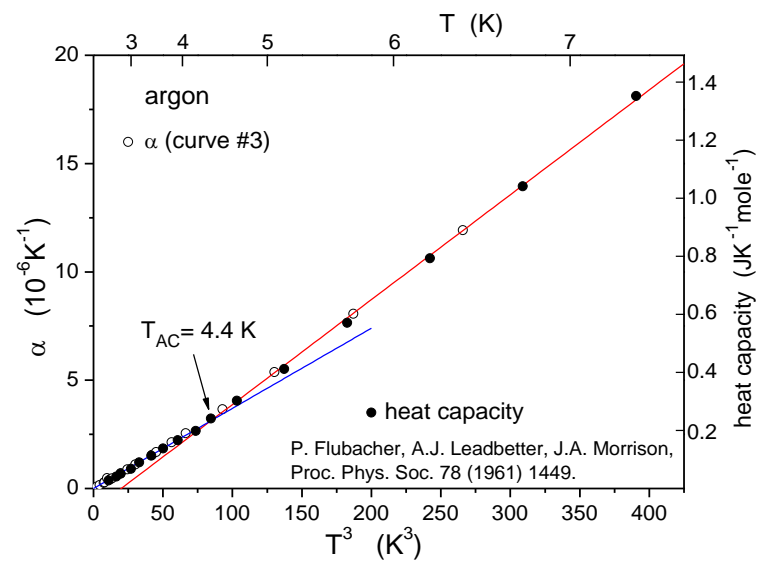

Figure 4. Coefficient of linear thermal expansion, $\alpha$, (circles) and heat capacity (filled circles) of the Rare Gas Solid argon as a function of absolute temperature to a power of three [21,22]. Data of both quantities are proportional to each other (Grüneisen relation) and have been matched together. The amplitude crossover at $T_{A C}=4.4$ $K$ marks the sharp onset of phonon contributions to the heat capacity. For $T<T_{A C}$ phonons contribute negligibly to the heat capacity.

It can be seen in Figure 4 that emergence of phonon contributions to the heat capacity proceeds as a crossover event, giving rise to a sharp kink (amplitude crossover). The increased pre-factor of the $T^{3}$ function for $T>T_{A C}$ is a qualitative measure for the heat capacity of the phonons. 
After a second crossover event at $T^{*}$ (not shown in Figure 4) the heat capacity increases much weaker and starts saturating towards Dulong-Petit limit. For $T>T^{*}$ Debye bosons contribute negligibly to the observed heat capacity. In other words, when phonons are the relevant excitations thermal conductivity tends to zero.

\section{Thermal Conductivity of Insulators}

Figure 5 shows two typical examples of the sharp maximum of thermal conductivity of insulators [2]. Amplitude crossover at $T=T_{A C}$ is on the high temperature flank of conductivity maximum. As a conclusion, upon emergence of phonons, heat transport by Debye bosons breaks down dramatically. In other words, phonons impede thermal conductivity by Debye bosons. Above a second crossover temperature at $T^{*}$ phonons are the relevant excitations and thermal conductivity tends to zero. This shows that thermal conductivity by phonons is negligibly small. Appearance of the maximum of thermal conductivity at a temperature of lower than $T_{A C}$ can be explained by damping processes in the propagation of Debye bosons and/or by the fact that the crossover at $\mathrm{T}_{\mathrm{AC}}$ is not an as sharp event as a phase transition but spreads over a finite width.

Another frequently occurring exponent is 2 (Figure 9). Comparison of Figure 7 and Figure 8 shows that depending on crystal quality $T^{3}$ and $T^{5 / 2}$ functions can occur for different $\mathrm{LiF}$ and $\mathrm{Al}_{2} \mathrm{O}_{3}$ samples.

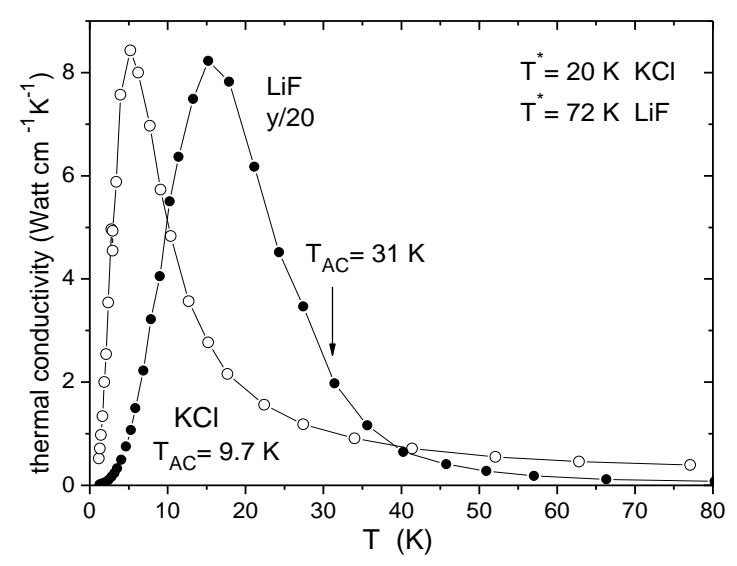

Figure 5. Two typical examples of the sharp maximum in thermal conductivity of insulators [2]. The maximum is in the temperature range $T<T_{A C}$ where Debye bosons (sound waves) are the relevant excitations and heat capacity exhibits $T^{3}$ dependence (Figure 4). For $T>T_{A C}$ phonons contribute finitely to heat capacity and thermal conductivity tends to zero. For explanation of $T_{A C}$ and $T^{*}$ see text.

In many materials the increasing and decreasing flank of thermal conductivity can be fitted by power functions of absolute temperature with positive and negative rational exponents, respectively. Such a functional change is typical for a crossover event. Figure 6 shows as an example thermal conductivity data of $\mathrm{NaF}$ [23]. $\mathrm{NaF}$ is perhaps the material with the highest occurring peak of thermal conductivity. The increasing flank of thermal conductivity can be fitted by the $\mathrm{T}^{3}$ function of the heat capacity of the Debye bosons. Limiting the fit range appropriately results in an exponent of $3.002 \pm 0.049$. The exponent of three shows, that the mean free path of the Debye bosons is larger than the linear dimension of the sample. Thermal conductivity then is given by the heat capacity of the Debye boson field. However, in thermal conductivity the $T^{3}$ function holds up to a lower temperatures than in heat capacity. Note that in the heat capacity the $T^{3}$ function holds up to $T_{A C}$ (Figure 4). We can assume that in the extremely good thermal conductor $\mathrm{NaF}$ damping processes for the Debye bosons are essentially of the intrinsic type due to interactions of Debye bosons with phonons. The decreasing flank of thermal conductivity can be fitted over a large temperature range by $\mathrm{T}^{-6}$ function.

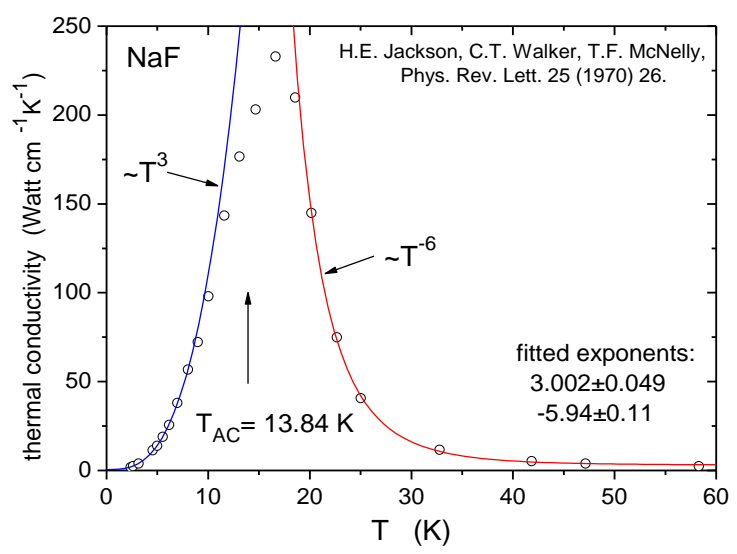

Figure 6. Increasing and decreasing flank of thermal conductivity maximum can be fitted by power functions of absolute temperature. This characterizes the maximum as crossover event. The crossover is from Debye bosons to phonons. When phonons are relevant thermal conductivity tends to zero. $T_{A C}=13.84 \mathrm{~K}$ is the crossover temperature identified in the heat capacity (see Figure 4) [23].

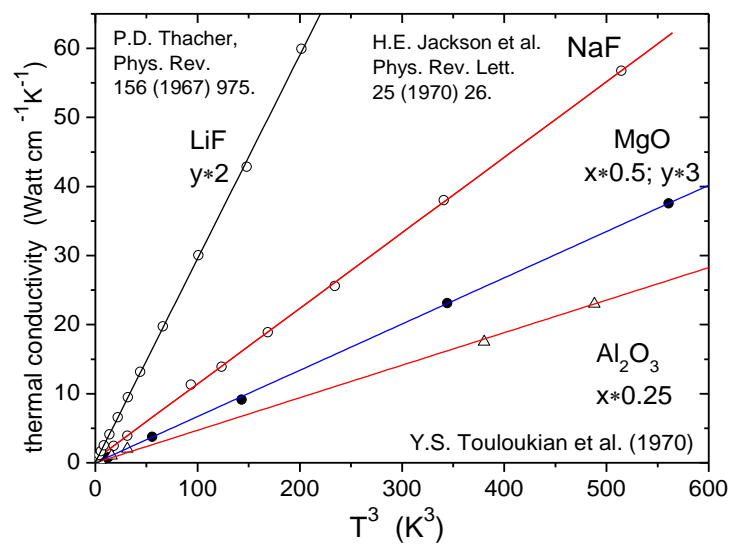

Figure 7. In hard materials with high crystal perfection thermal conductivity starts by the $T^{3}$ function of the heat capacity of the Debye boson field [2,23,24]. Condition for $T^{3}$ dependence is that the mean free path of the Debye bosons is larger than the linear dimension of the sample.

In hard materials with high crystal perfection thermal conductivity commonly starts by $T^{3}$ function (Figure 7). For $\mathrm{LiF}$ the fitted exponent is $2.988 \pm 0.024$ [24]. For crystals with higher defect concentration propagation of Debye bosons becomes damped. When the mean free path becomes shorter than the linear dimension of the sample, the exponent falls below the value of three (Figures 7-9). Sound velocities and thermal conductivity then are sample 
dependent. This makes measurements of thermal conductivity, elastic constants, i.e. sound velocities badly reproducible [6]. Thermal conductivity then starts by a power function of temperature with rational exponent of lower than three. The fact that rational exponents can be identified proves definite damping mechanisms. Change of exponent is a threshold controlled event. Figure 8 shows examples for the exponent of 2.5. The mean free path of the Debye bosons therefore can be assumed to be $\sim T^{0.5}$.

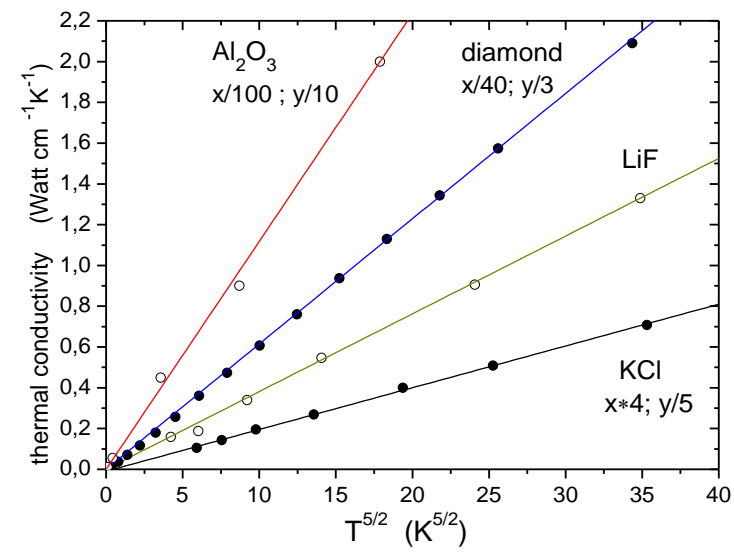

Figure 8. For crystals with higher defect concentration thermal conductivity can start by $T^{5 / 2}$ function [2]. The mean free path of the Debye bosons therefore is $\sim T^{-0.5}$.

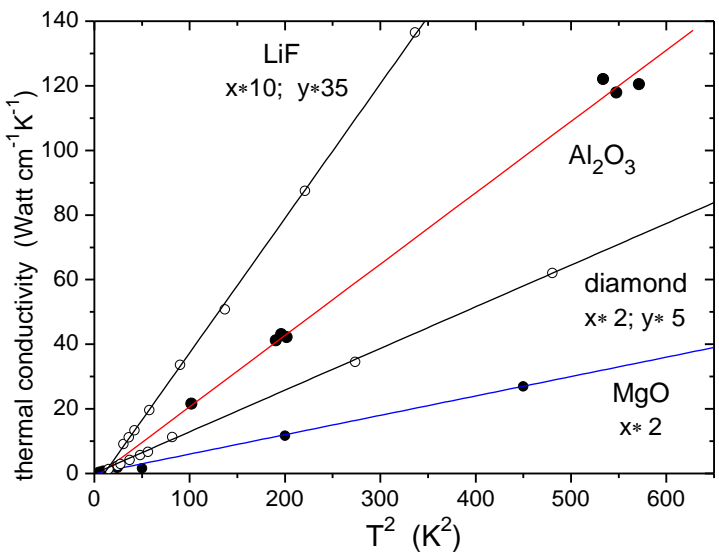

Figure 9. For crystals with a fairly high concentration of lattice defects, thermal conductivity can start by $T^{2}$ function [2]. The mean free path of the Debye bosons therefore is $\sim T^{-1}$.

\section{Thermal Conductivity of Metals}

In metals thermal conductivity is exclusively due to electronic degrees of freedom. The $\mathrm{T}^{3}$ term due to Debye bosons is completely absent in the thermal conductivity data of the metals. This is not a matter of different absolute thermal conductivity values of metals and of insulators (compare Figure 7 and Figure 10). Note that at low temperatures, thermal conductivity of insulators with high crystal perfection can be much larger than for metals (Figure 12). As is well known, at low temperatures thermal conductivity of all metals starts as a linear function of temperature, independent of crystal quality (Figure 10) [3]. Agreement with the linear temperature dependence of the electronic heat capacity indicates that in metals the mean free path of the carriers of heat transport must be very large.

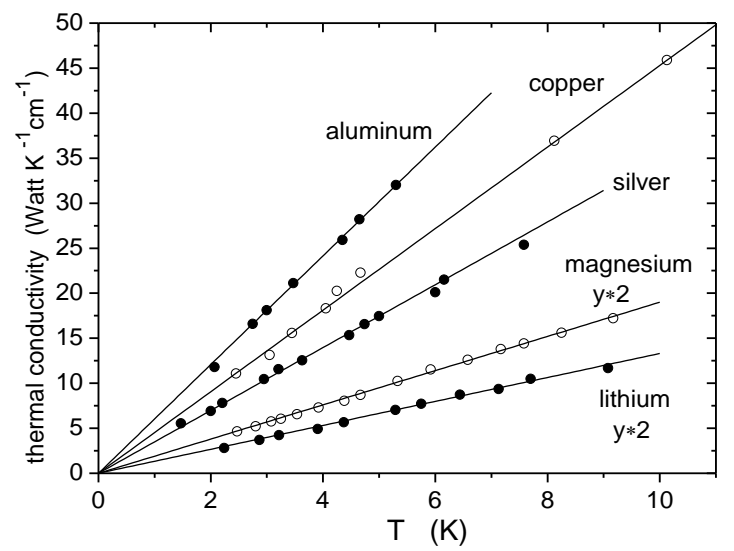

Figure 10. Thermal conductivity of metals starts as the heat capacity of the $C B$ bosons as a linear function of temperature [3]. This indicates that the mean free path of the $C B$ bosons must be very large. Note that the $T^{3}$ term due to Debye bosons is completely absent (compare Figure 7).

Linear temperature dependence of the electronic heat capacity seems to be in accord with conventional band theories [25]. This, however, is fortuitous. According to the band theories the linear-in-T electronic heat capacity should persist up to a considerable fraction of Fermi temperature, possibly modified by a small additional term quadratic in temperature. This is not confirmed experimentally. It turns out that for temperatures of larger than $\sim 300 \mathrm{~K}$ where the lattice heat capacity increases only slowly as a function of temperature the linear-in- $T$ electronic heat capacity can no longer be identified and, as a consequence, must have largely disappeared. This is as for the $T^{3}$ function of the heat capacity of the Debye bosons, which is completely absent for temperatures of larger than crossover temperature at $10 \ldots 30 \mathrm{~K}$. Subtracting from the experimental high-temperature heat capacity data of the metals [26] the linear electronic heat capacity as evaluated at low temperatures [27], results in a decreasing heat capacity as a function of increasing temperature (Figure 11). This unphysical result shows that the linear-in-T electronic heat capacity must pass a maximum (crossover) and afterwards falls to zero. Qualitatively this behavior conforms to the heat capacity of the Debye boson field which also has dropped down to a very small value at ambient temperature. Otherwise the heat capacity of all solids, including metals, would not saturate reasonably at atomistic Dulong-Petit limit. As a conclusion, low temperature electronic heat capacity and low temperature thermal conductivity must be due to bosons. Apparently, the conduction band has properties as a spatially continuous (translational invariant) medium with freely propagating bosons as low temperature excitations. The bosons could be charge density waves [28]. We will call them CB bosons. From the large mean free path of the $\mathrm{CB}$ bosons it becomes evident that the $\mathrm{CB}$ bosons cannot have charge. In contrast to the linear dispersion of the Debye bosons the dispersion of the CB bosons is completely unknown. Presumably, the $\mathrm{CB}$ bosons have no mass and therefore have linear dispersion. 


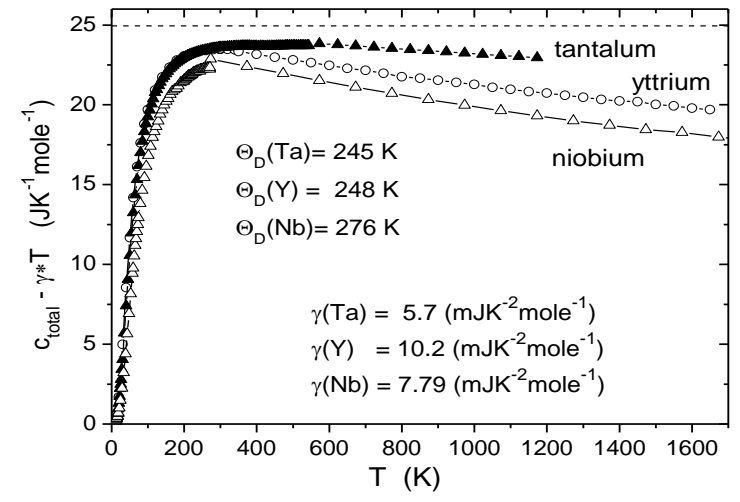

Figure 11. After subtraction from the high temperature heat capacity of the metals [26] the linear-in-T electronic heat capacity of $c_{e}=\gamma \cdot T$ observed at low temperatures [27] a decreasing temperature dependence results. This shows that at high temperatures the electronic heat capacity must be much smaller than $c_{e}=\gamma \cdot T$.

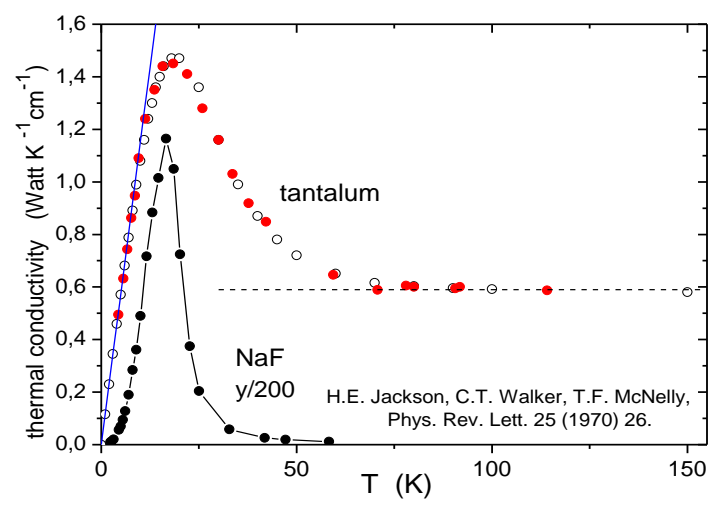

Figure 12. Typical difference between thermal conductivity of an insulator $(\mathrm{NaF})$ and of a metal $(\mathrm{Ta})[3,23]$. For both classes of materials the maximum is at a similar temperature but is due to different types of bosons. In metals thermal conductivity is entirely electronic and starts as $\sim T$. In insulators it is due to Debye bosons and starts as $\sim T^{3}$. Heat transport by phonons is negligible. The nearly constant high-temperature thermal conductivity of the metals is due to the conventional electronic band states.

As Figure 10 shows, in the thermal conductivity of the metals Debye's $T^{3}$ function is completely absent. This is surprising since in the heat capacity the $T^{3}$ function can clearly be identified. Superposition of the terms $\sim T$ and $\sim T^{3}$ in the low temperature heat capacity of the metals indicates that $\mathrm{CB}$ bosons and Debye bosons interact very weakly. In other words, $\mathrm{CB}$ bosons are nearly not coupled to the elastic degrees of freedom. This conforms to their large mean free path. Note that this is different in ordered magnets. In ordered magnets the heat capacity contributions of Debye bosons and of Goldstone bosons appear alternatively in the heat capacity [29]. This shows that the two boson types interact significantly. In fact, as the phenomenon of magnetostriction shows, elastic and magnetic degrees of freedom are coupled.

Complete unimportance of Debye bosons (and phonons) for heat transport in metals shows that there must be a symmetry selection rule deciding which boson type is relevant for thermal conductivity. This seems to be a matter of which boson type has the largest mean free path.
It is reasonable to identify the maximum of thermal conductivity of the metals (Figure 12) with the assumed maximum of the heat capacity of the $\mathrm{CB}$ bosons. For temperatures above maximum the heat capacity of the $\mathrm{CB}$ bosons can be assumed to fall to a very small value. However thermal conductivity assumes a rather low but finite and nearly temperature independent value. In this temperature range atomistic dynamics prevails, and band theories might be correct [25,27]. It is evident that the calculated pre-factor of the linear-in- $T$ electronic heat capacity of the band theories must be much smaller than the observed low temperature value $(\gamma)$. Fair agreement of the observed low temperature $\gamma$-values with the $\gamma$-values calculated for the free electron gas is much surprising and physically hard to understand [27]. At high temperatures the heat capacity of the conventional conduction band states can be assumed to be $\sim T$ but with a pre-factor of much smaller than for the CB bosons. Since the high temperature thermal conductivity of metals is constant the scattering probability for the conventional band electrons should be $\sim \mathrm{T}$. As a conclusion, in contrast to $\mathrm{CB}$ bosons the atomistic carriers of heat transport -the conventional band states- are coupled to the lattice. Note that at room temperature thermal conductivity of metals is much larger than thermal conductivity of insulators. However, for insulators with a high crystal perfection thermal conductivity at maximum can be larger by two orders of magnitude compared to the conductivity maximum of metals (see Figure 12).

\section{Conclusions}

Heat transport in solids proceeds independent of the atomistic structure. It is common to call this type of behaviour universal. Quite generally, whenever thermodynamic observables show universality (independence of lattice structure), the dynamics is controlled by a boson field. Universality results because the bosons propagate ballistic, independent of lattice structure. For instance, universality is well known to hold for spin dynamics in the vicinity of the magnetic ordering transitions. As was shown by $\mathrm{RG}$ theory [7,8], on approaching the magnetic ordering temperature from the paramagnetic side, spins and interactions between spins become of no importance on the critical spin dynamics. Spin dynamics is as for a continuous medium. Note that in a continuous medium there are no atoms or spins. Since the excitations of a continuous medium are bosons, the universal critical spin dynamics is controlled by a boson field [30,31]. In [29] it could be shown that the bosons of the magnetic continuum are essentially magnetic dipole radiation generated by the processing spins. Only sufficiently above ordering temperature spin dynamics is determined by local exchange interactions between individual pairs of spins. Change from exchange defined dynamics to boson defined dynamics is a crossover. The crossover occurs at a distinguished temperature above ordering transition.

In diamagnetic solids a quite analogous crossover from atomistic dynamics to boson dynamics occurs on approaching $\mathrm{T}=0$. In other words, the functionality of the magnetic ordering transition has shifted to $T \rightarrow 0$. In this sense $\mathrm{T}=0$ has the character of a critical temperature. Quite generally, in the vicinity of critical temperatures the dynamics seems to be determined by a boson field. However, in most cases the bosons are not yet identified. This applies to the bosons of the conduction band continuum in metals as well. We have called these bosons 
CB bosons. Fortunately, the bosons of the elastic continuum are well known from practical experience as Debye bosons or sound waves. As we now know, the bosons of the continuous solid have a different excitation spectrum compared to the atomistic excitations of phonons (Figures 2 and 3). In insulators, thermal conductivity is exclusively by Debye bosons, and reaches a maximum at about $10 \ldots 30 \mathrm{~K}$. In this temperature range phonons are negligible (not relevant). For larger temperatures phonons become the relevant excitations and thermal conductivity decreases dramatically. In the temperature dependence of the observed heat capacity, the crossover of thermal energy from boson field to lattice system appears as gradual change between concave up and concave down curvature. Thermal conductivity allows for a much clearer visualization of this crossover. This is because heat transport by phonons is negligibly small in insulators and in metals as well. As a consequence, thermal conductivity drops to zero when phonons are the relevant excitations. In metals thermal conductivity is exclusively due to the electronic degrees of freedom. Surprisingly, in spite of completely different boson types, in metals and in insulators the maxima of thermal conductivity are at comparable temperatures $(10 \ldots 30 \mathrm{~K})$.

\section{References}

[1] P. G. Klemens, "Theory of the Thermal Conductivity of Solids", in: R. P. Tye (Ed.), Thermal Conductivity vol. 1, Academic Press, London and New York, 1-68, 1969.

[2] Y. S. Touloukian, R. W. Powell, C. Y. Ho, P. G. Klemens, Thermophysical Properties of Matter, Vol. 2, Thermal Conductivity of Nonmetallic Solids, IFI/Plenum, New York-Washington, 1970.

[3] Y. S. Touloukian, R. W. Powell, C. Y. Ho, P. G. Klemens, Thermophysical Properties of Matter, Vol.1, Thermal Conductivity of Metallic Elements and Alloys, IFI/Plenum, New York-Washington, 1970.

[4] G. Leibfried, "Gittertheorie der mechanischen und thermischen Eigenschaften der Kristalle", in: S. Flügge (Ed.), Handbuch der Physik, Vol.7, part 1, Berlin: Springer, 104-324, 1955.

[5] M. Born, K. Huang, Dynamical Theory of Crystal Lattices, Oxford: Clarendon Press, 1956.

[6] R. F. S. Hearmon in: K.-H. Hellwege, A.M. Hellwege (Eds.), Landolt-Börnstein, vol. III/11, Berlin: Springer, 1-286, 1979.

[7] K. G. Wilson, J. Kogut, "The Renormalization Group and the $\varepsilon$ Expansion”, Phys. Rep. 12C, 75-199, 1974.

[8] K. G. Wilson, "The renormalization group: Critical phenomena and the Kondo problem", Rev. Mod. Phys. 47, 773-840, 1975.

[9] G. A. Alers, "Use of Sound Velocity Measurements in Determining the Debye Temperature of Solids", in: Physical Acoustics, ed. by W.P. Mason, Vol. III-part B, Academic Press, 1-42, 1965.

[10] J. C. Ho, D.P. Dandekar, "Low-temperature heat capacities of $\mathrm{RbCl}, \mathrm{RbBr}$ and $\mathrm{CsCl}$ ”, Phys. Rev. B 30, 2117-2119, 1984.

[11] W. T. Berg, J. A. Morrison, "The thermal properties of alkali halide crystals", Proc. Roy. Soc. London A242, 467-477, 1957.
[12] U. Köbler, "On the Distinction between Debye Bosons and Acoustic Phonons", Int. J. Therm. 18, 277-284, 2015.

[13] K. H. Hellwege, Einführung in die Festkörperphysik. $3^{\text {th }} E d$., Berlin: Springer, 1988.

[14] R. N. Claytor, B. J. Marshall, "Specific Heat and Elastic Constants of Sodium Iodide at Low Temperatures“, Phys. Rev. 120, 332-334, 1960.

[15] P. Debye, "Zur Theorie der spezifischen Wärmen”, Ann. Physik 39, 789-839, 1912.

[16] I. Barin, Thermochemical Data of Pure Substances, Weinheim: VCH, 1995.

[17] R. Cowley, W. Cochran, B. N. Brockhouse, A. D. B. Woods, "Lattice Dynamics of Alkali Halide Crystals. III. Theoretical”, Phys. Rev. 131, 1030-1039, 1963.

[18] M. J. L. Sangster, G. Peckham, D. H. Saunderson, "Lattice dynamics of magnesium oxide", J. Phys. C 3, 1026-1036, 1970.

[19] T. H. K. Barron, W. T. Berg, J. A. Morrison, "On the heat capacity of crystalline magnesium oxide", Proc. Roy. Soc. (London) A250, 70-83, 1959.

[20] T. H. K. Barron, G. K. White, Heat Capacity and Thermal Expansion at Low Temperatures, Kluwer, Dortrecht, 1999.

[21] Y. S. Touloukian, R. K. Kirby, R. E. Taylor, T. Y. R. Lee, Thermophysical Properties of Matter, vol.13, Thermal Expansion of Nonmetallic Solids, New YorkWashington: IFI/Plenum, 1977.

[22] P. Flubacher, A. J. Leadbetter, J. A. Morrison, “A Low Temperature Adiabatic Calorimeter for Condensed Substances, Thermodynamic Properties of Argon", Proc. Phys. Soc. 78, 1449-1461, 1961.

[23] H. F. Jackson, C. T. Walker, T. F. McNelly, "Second Sound in NaF", Phys. Rev. Lett. 25, 26-28, 1970.

[24] P. D. Thacher, "Effect of Boundaries and Isotopes on the Thermal Conductivity of LiF", Phys. Rev. 156, 975988, 1967.

[25] A. Sommerfeld, H. Bethe, Elektronentheorie der Metalle, Berlin: Springer, 1967.

[26] Y. S. Touloukian, E. H. Buyco, Thermophysical Properties of Matter, vol.4, Specific Heat of Metallic Elements and Alloys, New York-Washington: IFI/Plenum, 1970.

[27] C. Kittel, Introduction to Solid State Physics, $7^{\text {th }}$ Ed., New York: John Wiley \& Sons, 157, 1996.

[28] G. Grüner, "The dynamics of charge-density waves", Rev. Mod. Phys. 60, 1129-1181, 1988.

[29] A. Hoser, U. Köbler, "Boson Fields in Ordered Magnets”, Acta Phys. Pol. A127, 350-352, 2015.

[30] U. Köbler, "Bosons and Magnons in Ordered Magnets", Acta Phys. Pol. A127, 1694-1701, 2015.

[31] A. Hoser, U. Köbler, "Functional crossover in the dispersion relations of magnons and phonons", J. Phys. Conf. Series 746, 012062, 1-8, 2016. 